\title{
USO DE IMAGENS DE MÉDIA RESOLUÇÃO ESPACIAL PARA O MONITORAMENTO DE DOSSÉIS DE Eucalyptus grandis
}

\author{
Use of medium spatial resolution images for monitoring of canopies of Eucalyptus grandis
}

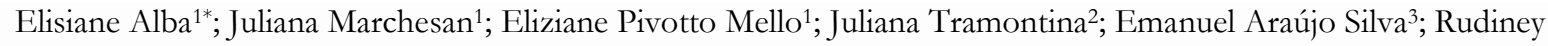 \\ Soares Pereira ${ }^{4}$

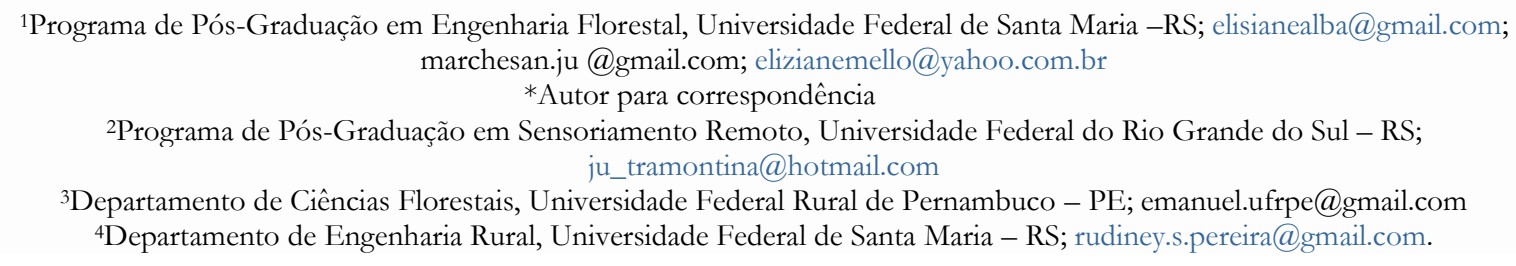

Artigo enviado em 21/04/2017, aceito em 22/09/2017 e publicado em 20/12/2017.

\begin{abstract}
Resumo - O objetivo desse estudo foi identificar plantios comerciais de Eucalyptus grandis com diferentes idades e estimar o volume total de madeira utilizando dados radiométricos do sensor OLI/Landsat 8. Demarcaram-se parcelas em povoamentos aos 4, 18 e 23 anos de idade, nas quais foi mensurado o volume total $\left(\mathrm{m}^{3} / \mathrm{ha}\right)$. A imagem OLI/Landsat 8 foi convertida para reflectância de superfície utilizando a correção radiométrica e, posteriormente gerados os índices de vegetação ARVI, DVI, GNDVI, MVI, NDVI, SAVI $\mathrm{L}=0,25$, $\mathrm{SAVI}_{\mathrm{L}=0,50}$ e SR. A diferenciação da idade dos dosséis foi estabelecida baseando-se na reflectância das bandas B4 e B5. Os dados espectrais e o volume total de madeira foram submetidos à análise de correlação e regressão múltipla baseada no método Stepwise. Os povoamentos foram diferenciados uma vez que o aumento da idade ocasionou a redução da reflectância na banda B5 e aumento na banda B4. Para a estimativa do volume total, apenas os povoamentos aos 4 anos de idade apresentaram bom ajuste, sendo o modelo composto pelo índice SAVI, explicando $74 \%$ da variabilidade do volume de madeira. Os dados espectrais extraídos de imagens OLI/Landsat 8 mostraram-se sensível às variações das características dos indivíduos nas diferentes fases de crescimento dos povoamentos, assim como na estimativa das variações do volume total.
\end{abstract}

Palavras-chave - Sensoriamento remoto, índices de vegetação, volume estimado.

\begin{abstract}
The objective of this study was to identify commercial Eucalyptus grandis plantations with different ages and estimate the total wood volume using OLI/Landsat 8 radiometric data. Plots were demarcated in stands at 4, 18 and 23 years of age, in which it was measured total volume $\left(\mathrm{m}^{3} / \mathrm{ha}\right)$. The OLI/Landsat 8 image was converted to surface reflectance using the radiometric calibration and, subsequently generated the vegetation indices ARVI, NDVI, NDVI, MVI, NDVI, $\mathrm{SAVI}_{\mathrm{L}=0.25} \mathrm{SAVI}_{\mathrm{L}=0.50}$ and $\mathrm{SR}$. The differentiation of the age of the canopies was established based on the reflectance of the B4 and B5 bands. The spectral and total volume of wood were subjected to correlation analysis and adjustment of the data to the regression models, leading to selection of variables through the Stepwise - Forward method. The stands were differentiated since the increase of the age caused the reduction in reflectance on B5 band and increase in band B4. For the estimation of total volume, only the stands at 4 years of age presented good adjustment, being the model composed by the SAVI index, explaining $74 \%$ of the volume variability of wood. The spectral data extracted from OLI/Landsat 8 images showed to be sensitive to variations in the characteristics of individuals in different growth stages of the stands, as well as in the estimation of changes in the total volume.
\end{abstract}

Keywords - Remote sensing, vegetation indices, estimated volume.

\section{INTRODUÇÃO}

A silvicultura vem ocupando grandes extensões no território nacional e segundo o boletim informativo Ibá
(2017), em 2016 os plantios florestais ocupavam uma área de 7,8 milhões de hectares, apresentando um crescimento de $0,5 \%$ em relação ao indicador de 2015 , sendo que esse aumento é exclusivo do crescimento das áreas de eucalipto. 
Entretanto, a área ocupada pelo setor florestal ainda é pouco expressiva quando comparada a área de produção agrícola, a qual corresponde aproximadamente a 75 milhões de hectares (IBGE, 2017). Assim, a agricultura representa $90,6 \%$ da área cultivada no Brasil, enquanto que o setor florestal corresponde a $9,4 \%$ desse total.

A observação regular do estado da floresta, incluindo a diferenciação da idade dos povoamentos e o monitoramento dos possíveis danos causados por deficiências nutricionais, estresse hídrico ou doenças, facilitam o desenvolvimento de estratégias e manejo adequado para cada situação (TILLACK et al., 2014), garantindo maior produtividade ao longo de seu desenvolvimento.

Com o avanço das técnicas de processamento digital de imagens e a crescente disponibilidade de dados de sensoriamento remoto, originou-se métodos indiretos de estimativas dos parâmetros da vegetação como o volume. Essas técnicas são utilizadas em conjunto com as informações obtidas em campo, de modo a subsidiar inventários florestais futuros, auxiliando na alocação de parcelas na área e reduzindo os custos de operação.

A partir de imagens orbitais, utiliza-se dos valores de reflectância espectral para a caracterização de povoamentos florestais como a diferenciação da idade dos povoamentos (AARDT; NORRIS 2008; PONZONI et al., 2015; GOERGEN et al. 2016) e estimativas de variáveis descritivas da vegetação tais como volume total (BERRA et al., 2013; COSTA et al., 2015), biomassa (FERRAZ et al., 2014), índice de área foliar (DUBE; MUTANGA, 2015), entre outros.

Goergen (2014) ao utilizar dados TM/Landsat 5 para estimativa do volume de povoamentos de eucalipto, obteve modelos que responderam até $54 \%$ da variabilidade dos dados de campo. Berra (2013) estimando o volume de $P$. elliottii com dados TM/Landsat 5 observou um maior ajuste, obtendo um coeficiente de determinação $\left(\mathrm{R}^{2}\right)$ de 0,70 .

Atualmente há inúmeros sistemas sensores disponíveis com características espaciais, temporais e espectrais diferenciadas. Entretanto, imagens com um nível de detalhamento maior, normalmente estão associadas a um alto custo de aquisição e menor área imageada pelo sensor, o que restringe seu uso em análises de áreas extensas e que demandam maior periodicidade.

Nesse sentido, a resposta espectral obtida por meio de produtos da série Landsat tem representado uma fonte de dados de fácil aquisição e gratuita. Inúmeras pesquisas relacionaram com êxito essas imagens multiespectrais com as características dos dosséis utilizando os sensores TM/Landsat 5, ETM+/Landsat 7 (BERRA, 2013; FERRAZ et al., 2014; COSTA et al., 2015; DUBE; MUTANGA, 2015; PONZONI et al., 2015; GOERGEN et al., 2016). Da série Landsat, atualmente apenas o satélite Landsat 8 dispõe dados atuais da superfície terrestre, dando sequência ao programa de observação da terra.
No entanto, esse novo satélite apresenta modificações, destacando-se o aumento da resolução radiométrica e o número de bandas espectrais. A mudança na resolução radiométrica de 8 bits para 16 bits proporcionou a distribuição dos níveis de cinza em uma escala maior (DUBE; MUTANGA, 2015), aumentando a capacidade de diferenciação entre alvos da superfície terrestre.

O objetivo desse estudo foi identificar plantios comerciais de Eucalyptus grandis com diferentes idades e estimar o volume total de madeira utilizando dados radiométricos do sensor OLI/Landsat 8 .

\section{MATERIAL E MÉTODOS}

A área de estudo está localizada entre as latitudes $32^{\circ}$ $33^{\prime} 22^{\prime \prime}$ a $32^{\circ} 41^{\prime} 03^{\prime \prime}$ Sul e longitudes $52^{\circ} 30^{\prime} 29^{\prime \prime}$ a $52^{\circ} 25^{\prime}$ 20" Oeste. A propriedade pertence a empresa Trevo Florestal LTDA, situada no município de Rio Grande no estado do Rio Grande do Sul, possuindo aproximadamente 5.800 hectares de áreas reflorestadas com as espécies de Pinus elliottii e Eucalyptus grandis (Figura 1).

A área de estudo apresenta solos originados de sedimentos quaternários do tipo Neossolo Quartzarênico, apresentando características predominantemente arenosas, mal drenadas e sobre relevo plano em toda sua extensão, com elevação de alguns metros em relação ao nível do mar. Baseando-se em análise dos dados SRTM (Shuttle Radar Topography Mission), observou-se que a área de estudo apresenta altitudes variando de 10 a $20 \mathrm{~m}$.

Esse estudo baseou-se nos dados de inventário florestal realizado no período de agosto a setembro de 2013 em povoamentos aos 4, 18 e 23 anos de idade. O inventário florestal foi desenvolvido com base na amostragem sistemática com a alocação das unidades amostrais (UAs) a cada 5 hectares, desse modo foram instaladas 53 UAs em povoamentos de Eucalyptus grandis, sendoque destas, $26 \mathrm{UAs}$ foram em povoamentos aos 4 anos de idade, 30 UAs aos 18 anos e 29 UAs aos 23 anos.

O volume total desses povoamentos foi obtido por meio de equação alométrica (Equação 1).

$$
\mathrm{V}=\left(\sum_{\mathrm{i}=1}^{\mathrm{n}} \pi \frac{\mathrm{d}_{\mathrm{i}}^{2}}{40000}\right) * \mathrm{~h}_{\mathrm{i}} * f
$$

Em que: $V$ é o volume total $\left(\mathrm{m}^{3} / \mathrm{ha}\right) ; \pi$ é o valor de PI $(3,141593)$; hi corresponde à altura da arvore i; $f$ é o fator de forma.

Os pontos amostrais apresentaram formato retangular com área de $420 \mathrm{~m}^{2}$ (20 x $\left.21 \mathrm{~m}\right)$. O espaçamento inicial dos plantios foi de $3 \times 2 \mathrm{~m}$, entretanto, essa medida tende a sofrer alterações no decorrer do desenvolvimento devido as práticas de manejo como o desbaste. A posição geográfica das UAs foi obtida utilizando GPS (Global Positioning System) Garmin Etrex Legend ${ }^{\circledR}$ no sistema de coordenadas UTM datum WGS - 84. 

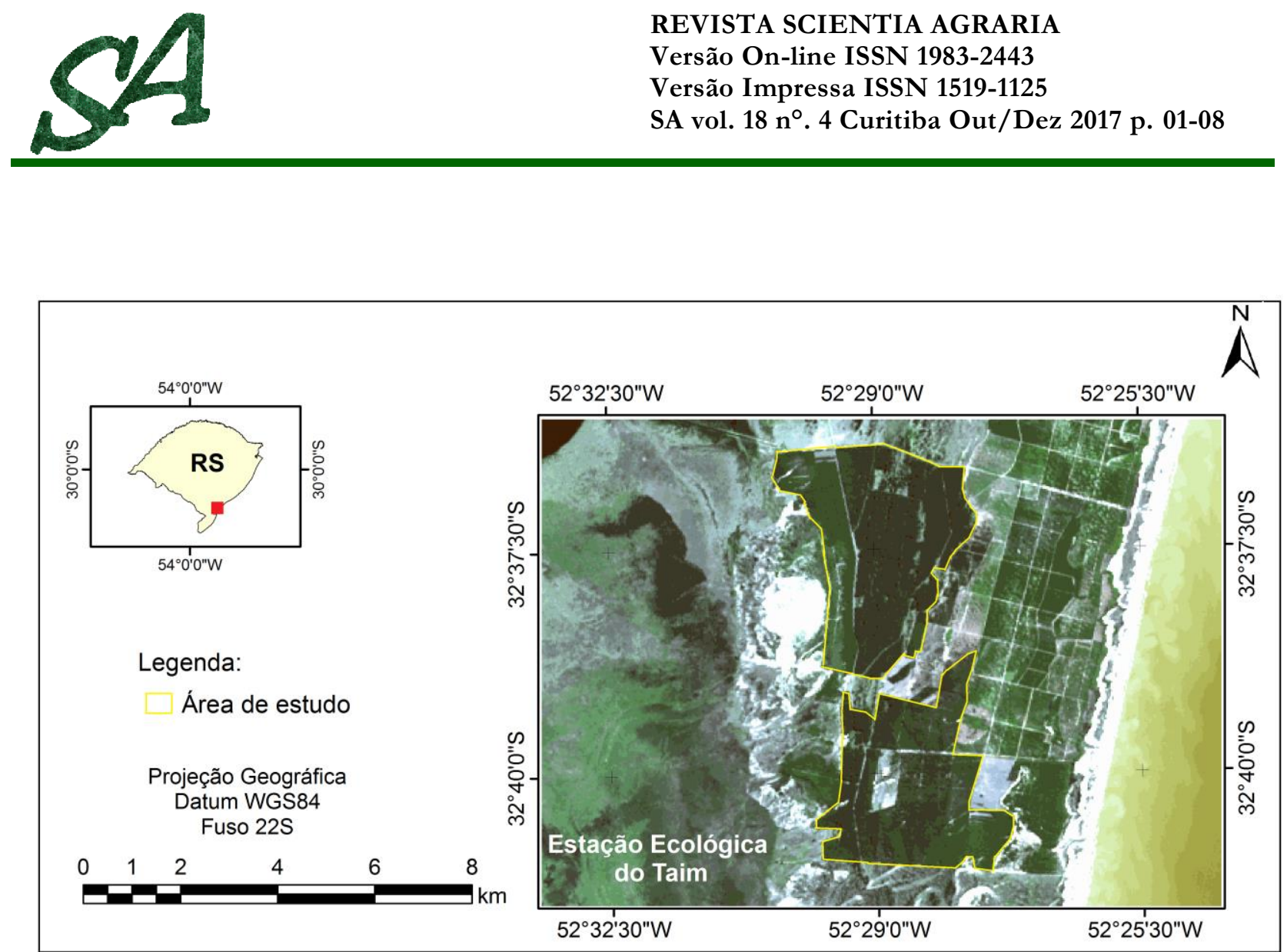

Figura 1 - Localização da área de estudo.

Os dados espectrais foram extraídos da imagem nas bandas $2(0,45-0,51 \mu \mathrm{m}), 3(0,53-0,59 \mu \mathrm{m}), 4(0,64-0,67$ $\mu \mathrm{m}), 5(0,85-0,88 \mu \mathrm{m}), 6(1,57-1,65 \mu \mathrm{m})$ e $7(2,11-2,29$ $\mu \mathrm{m})$ do sensor OLI/Landsat8 (órbita-ponto 221/083) datada em 30 de agosto de 2013. Essas faixas espectrais foram selecionadas por apresentarem maior resposta à vegetação.

De modo a tornar possível a obtenção dos parâmetros biofísicos dos povoamentos florestais, os Números Digitais (NDs) da imagem foram transformados em reflectância de superfície pelo aplicativo Environment for Visualizing Images (ENVI 5.1®), por meio do algoritmo Fast Line-of-sight Atmospheric Analysis of Spectral Hypercubes (FLAASH). Nesse processo a elevação média do terreno foi considerada de 15 $\mathrm{m}$, utilizando como modelo atmosférico "mid-latitude summer" e modelo de aerossol foi escolhido o "rural", atribuindo o valor de $100 \mathrm{~km}$ à visibilidade no momento do imageamento.

Após obter os valores de refletância de superfície, elaboraram-se índices de vegetação (Tabela 1). Os dados espectrais foram utilizados, inicialmente, na diferenciação dos estágios de crescimento de acordo com a idade dos povoamentos, baseando-se na resposta espectral das bandas do vermelho (B4) e infravermelho próximo (B5), representadas graficamente nas orientações $x$ e $y$, respectivamente.

A banda B5 do sensor OLI atua na faixa espectral de 850 a $880 \mathrm{~nm}$ do espectro eletromagnético, enquanto que a banda B4 compreende comprimento de onda de 640 a 670 nm. A caracterização dos dosséis baseou-se nestas faixas espectrais devido a maior interação com a vegetação conforme descrito na literatura (PONZONI, et al. 2012; JENSEN, 2009).

A fim de investigar a relação entre os dados espectrais e o volume total de madeira ( $\mathrm{m}^{3} / \mathrm{ha}$ ) aos 4,18 e 23 anos de idade, aplicou-se a análise de Correlação Linear de Pearson (r) e a análise de regressão múltipla para estimativa dessa variável. Na modelagem empírica, as bandas espectrais do sensor OLI e os índices de vegetação foram as variáveis independentes, enquanto que o volume total de madeira $\left(\mathrm{m}^{3} / \mathrm{ha}\right)$ foi a variável dependente. Nesse processo utilizouse o método Stepwise, sendo selecionadas as variáveis espectrais com maior contribuição ao modelo proposto.

Para a escolha do modelo que melhor se ajustou aos dados, procedeu-se com a avaliação do comportamento dos modelos matemáticos selecionados, levando em consideração os critérios estatísticos recomendados por Schneider et al. (2009): coeficiente de determinação ajustado $\left(\mathrm{R}^{2}{ }_{\mathrm{aj}}\right)$, erro padrão da estimativa $\left(\mathrm{S}_{\mathrm{yx}}\right)$, índice de Furnival (IF) e teste $\mathrm{F}$ com o nível de significância de $5 \%$ de probabilidade.

$\mathrm{Na}$ modelagem do volume foi considerado apenas modelos com ajuste igual ou superior a $70 \%\left(\mathrm{R}_{\mathrm{aj}} \geq 0,70\right)$, uma vez que modelos com ajuste inferior a esse percentual englobariam grandes erros nas estimativas e assim, não devem ser utilizados para predição dessa variável com dados espectrais obtidos por plataformas orbitais.

A extração das informações de reflectância dos pixels situados em cada unidade amostral, assim como a modelagem do volume foram realizadas em linguagem de programação R, versão 3.4.0 (R DEVELOPMENT CORE TEAM, 2016). 
Tabela 1 - Índices de Vegetação analisados no presente estudo.

\begin{tabular}{|c|c|c|}
\hline Índice de vegetação & Fórmula & Autor \\
\hline ARVI & ARVI $=\frac{\varrho_{\text {IVP }}-\left(2 * \varrho_{V}+\varrho_{\text {azul }}\right)}{\varrho_{I V P}+\left(2 * \varrho_{V}+\varrho_{\text {azul }}\right)}$ & Kaufman e Tanré (1992) \\
\hline DVI & $\mathrm{DVI}=\varrho_{\mathrm{IVP}}-\varrho_{\mathrm{V}}$ & Clevers (1988) \\
\hline GNDVI & GNDVI $=\frac{\varrho_{\text {IVP }}-\varrho_{\text {Verde }}}{\varrho_{\text {IVP }}+\varrho_{\text {Verde }}}$ & Sousa e Ponzoni (1998) \\
\hline MVI & MVI $=\frac{\varrho_{\text {IVP }}-\varrho_{\text {SWIR }}}{\varrho_{\text {IVP }}+\varrho_{\text {SWIR }}}$ & Sousa e Ponzoni (1998) \\
\hline NDVI & $\mathrm{NDVI}=\frac{\varrho_{\mathrm{IVP}}-\varrho_{\mathrm{V}}}{\varrho_{\mathrm{IVP}}+\varrho_{\mathrm{V}}}$ & Rouse et al. (1974) \\
\hline $\mathrm{SAVI}_{\mathrm{L}=0,25}$ & SAVI $=\frac{\left(\varrho_{\mathrm{IVP}}-\varrho_{\mathrm{V}}\right)}{\left(\varrho_{\mathrm{IVP}}+\varrho_{\mathrm{V}}+0,25\right)} *(1+0,25)$ & Huete et al. (1985) \\
\hline $\mathrm{SAVI}_{\mathrm{L}=0,50}$ & SAVI $=\frac{\left(\varrho_{I V P}-\varrho_{V}\right)}{\left(\varrho_{I V P}+\varrho_{V}+0,50\right)} *(1+0,50)$ & Huete et al. (1985) \\
\hline SR & $\mathrm{SR}=\frac{\varrho_{\mathrm{IVP}}}{\varrho_{\mathrm{V}}}$ & Jordan (1969) \\
\hline
\end{tabular}

ARVI: Atmospherically Resistant Vegetation Index; DVI: Difference Vegetation Index; GNDVI: Green Normalized Difference Vegetation Index; MVI: Moisture Vegetation Index; NDVI: Normalized Difference Vegetation Index; SAVI: Soil adjusted vegetation index; SR: Simple Ratio Vegetation Index; $\varrho_{\text {Azul }}=$ Reflectância da banda do azul; $\varrho_{\text {Verde }}=$ Reflectância da banda do verde; $\varrho_{\mathrm{V}}=$ Reflectância da banda do vermelho; $\varrho_{\mathrm{IVP}}=$ Reflectância da banda do Infravermelho próximo; $\varrho_{\text {SWIR }}=$ Reflectância da banda do infravermelho de ondas curtas; $\mathrm{L}=$ constante que minimiza os efeitos do solo, utilizada nesse estudo o valor de 0,25 e 0,50 .

A validação dos modelos selecionados foi desenvolvida pelo teste Qui-quadrado $\left(\mathrm{X}^{2}\right)$, ao nível de significância de $95 \%$ de probabilidade, utilizando $20 \%$ das UA. Assim, os dados foram divididos formando dois conjuntos, sendo um para a calibração e outro para a validação dos modelos.

\section{RESULTADOS E DISCUSSÃO}

Os povoamentos comerciais apresentaram comportamentos distintos em cada uma das idades estudadas, demonstrando que a resposta espectral é sensível ao estágio de desenvolvimento dos indivíduos (Figura 2). O comportamento espectral de uma vegetação sadia demonstra picos de maior reflectância na faixa do verde e infravermelho próximo, enquanto que nas demais faixas a energia é absorvida em maiores quantidades (PONZONI et al., 2012; JENSEN, 2009).

Entretanto, observou-se que com o aumento da idade dos povoamentos há a diminuição da reflectância na banda B5, que corresponde a faixa do infravermelho próximo e aumento da reflectância na banda B4, a qual consiste na faixa do vermelho do sensor OLI/Landsat 8 .

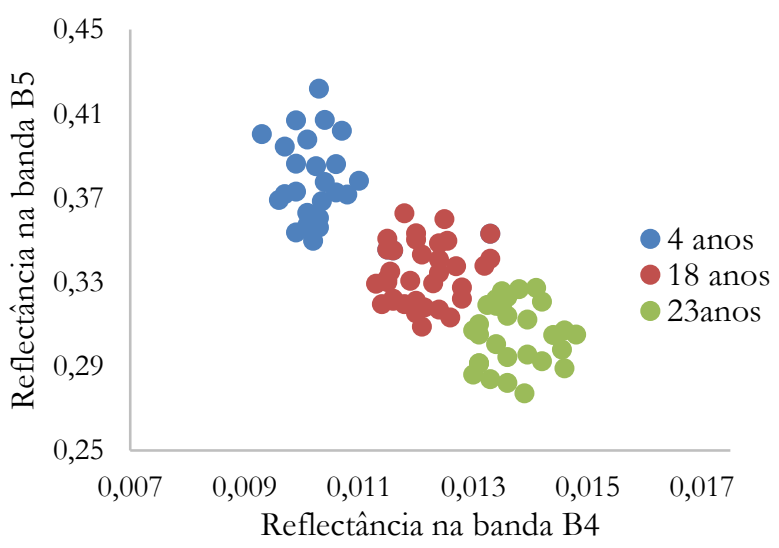

Figura 2. Resposta espectral dos povoamentos de $E$. grandis aos 4, 18 e 23 anos de idade em relação as bandas B4 e B5 do sensor OLI/Landsat 8.

A ausência de desbaste nos povoamentos aos 4 anos de idade propiciou maior cobertura de dossel, e com isso, menor influência do solo na reflectância. A medida que os povoamentos se tornam mais maduros, há maior influência 
do solo e de sombra, relacionando-se diretamente com os valores de reflectância. Carreiras et al. (2006) definem que a intensidade do sinal armazenado pelo sensor nas bandas do infravermelho próximo e vermelho também é fortemente relacionada com a classe de fundo, desse modo quanto maior a cobertura do dossel, maior a reflectância na faixa do infravermelho próximo devido a menor influência do brilho do solo na resposta espectral.

Segundo Ponzoni et al. (2015), fatores que explicariam o comportamento espectral dos dosséis seria a redução da influência das folhas verdes e aumento da participação de galhos secos depositados sobre o solo, especialmente quando realizado o desbaste dos povoamentos. Essa prática de manejo acumula grande quantidade de galhos e cascas no interior do povoamento.

$\mathrm{O}$ aumento do volume relaciona-se diretamente com o crescimento em diâmetro e altura dos indivíduos, e para povoamentos jovens, esse fator culmina na maior produção de folhas verdes, com elevada atividade fotossintética (Berra, 2013). Esse processo, segundo Ponzoni et al. (2012) faz com que povoamentos jovens apresentem valores elevados de reflectância na região do infravermelho próximo e menor na região do visível.

Nesse sentido, os resultados demonstraram que aos 4 anos de idades, a reflectância na banda B5 é superior àquela apresentada aos 18 e 23 anos. Os povoamentos jovens amostrados nesse estudo apresentam maior densidade populacional (ausência de desbaste) e consequentemente, maior cobertura da superfície do solo, aliado a um dossel formado por folhas jovens, sendo assim, esse conjunto de fatores justificam a maior intensidade do sinal nessa faixa espectral.

Por outro lado, observou-se aumento da reflectância na banda B4 com o aumento da idade, o que se atribui à redução da atividade dos pigmentos foliares, os quais utilizam a Radiação Eletromagnética (REM) incidente para processos fisiológicos da planta, como a fotossíntese. Desse modo, os povoamentos aos 23 anos apresentam taxa de crescimento inferior (capacidade fotossintética) que aos 18 e aos 4 anos de idade. Segundo Jensen (2009), a reflectância na região do visível (faixas do azul, verde e vermelho) é influenciada pela concentração de pigmentos foliares.

Neste contexto, Goergen et al. (2016) obtiveram resultados satisfatórios utilizando dados TM/Landsat 5 para a diferenciação das idades de povoamentos de Eucalyptus sp. aplicando técnicas de análise multivariada. Do mesmo modo, Aardt e Norris (2008) diferenciaram as classes de idade de povoamentos de Eucalyptus sp. e Acacia sp. a partir de dados do sensor hiperespectral Casi.

A Tabela 2 mostra os coeficientes de Correlação Linear de Pearson ( $r$ ) entre o volume total de madeira e as variáveis espectrais fortemente correlacionadas. As variáveis espectrais B5, DVI, NDVI, $\mathrm{SAVI}_{\mathrm{L}=0,25}$ e $\mathrm{SAVI}_{\mathrm{L}=0,50}$ melhor se correlacionam com os dados de campo. Esses resultados estão diretamente relacionados às condições geométricas definida no momento da aquisição da imagem pelo sensor OLI. A área de estudo possui relevo plano, minimizando os efeitos negativos da diferença de iluminação entre os pontos amostrais. A variável florestal apresentou melhor correlação com o índice $\mathrm{SAVI}_{\mathrm{L}=0,50}$ expressando um coeficiente aos 4, 18 e 23 anos de $-0,7895,-0,8331$ e 0,7063 , respectivamente. Na sequência, o índice DVI apresentou boa correlação nas três amostras observadas.

O índice SAVI, ao considerar a influência do solo na resposta espectral apresenta-se em diversos estudos como a variável de melhor correlação com os parâmetros florestais (CASSOL, 2013; COSTA et al., 2015). Os povoamentos aos 4 anos de idade, estão associados à alta densidade, conforme enfatizado anteriormente. Entretanto, nota-se que mesmo nesses povoamentos, há a influência do sub bosque na resposta espectral, sendo essa relação comprovada pelo maior ajuste do índice SAVI. Huete et al. (1985) avaliou a influência do solo na resposta espectral em relação a diferentes graus de cobertura, constatando que esse fator interfere não apenas em dosséis pouco densos, como também em dosséis com cobertura vegetal densa.

Do mesmo, em estudos desenvolvidos por Cassol (2013), Maciel (2002) e Bernardes (1998), o índice SAVI destacou-se como uma das variáveis espectrais de maior relação com a biomassa florestal de uma Floresta Ombrófila Mista, a qual apresenta grande densidade populacional. Esses autores reforçam que o do brilho do solo influencia na resposta espectral, mesmo em dosséis fechados e com grande densidade de indivíduos.

Ao analisar as bandas espectrais, observou-se que a banda B5 melhor se correlacionou com a variável florestal nas três idades de análise, apresentando $\mathrm{r}$ no valor de 0,7774, -0,8294 e 0,6802 aos 4, 18 e 23 anos, respectivamente. Esses resultados são consistentes com os encontrados por Berra (2013), Goergen (2014) e Pacheco et al. (2012). A faixa espectral compreendida pela banda B5 apresenta alta reflectância para a vegetação, o que proporciona maior sensibilidade às mudanças ocorridas na cobertura florestal, exibindo maior relação com as variáveis biofísicas.

A região do infravermelho próximo pode apresentar tanta relação inversa quanto direta para a vegetação, porém Puhr e Donoghue (2000) reportam a relação inversa à presença de sub bosque e/ou sombreamento, uma vez que a existência desses fatores influencia na redução dos valores de reflectância com o aumento da variável florestal, já que dossel com sombreamento ou com sub bosque apresentam menor reflectância na faixa do infravermelho próximo. Assim, para os povoamentos com idade de 4 e 18 anos, a banda B5 apresentou relação inversa e aos 23 anos grande parte das variáveis espectrais demonstraram relação direta, o que evidenciou uma associação variável, influenciada pela idade e características da cobertura do dossel. 
Tabela 2. Análise de Correlação Linear de Pearson entre o volume total $\left(\mathrm{m}^{3} / \mathrm{ha}\right)$ e a reflectância das bandas espectrais e índices de vegetação.

\begin{tabular}{|c|c|c|c|}
\hline \multirow{2}{*}{ Variável } & \multicolumn{3}{|c|}{$\mathrm{V}\left(\mathrm{m}^{3} / \mathrm{ha}\right)$} \\
\hline & 4 anos & 18 anos & 23 anos \\
\hline B5 & $-0,7774 * *$ & $-0,8294 * *$ & $0,6802^{* *}$ \\
\hline DVI & $-0,7741 * *$ & $-0,8325^{* *}$ & $0,6892^{* *}$ \\
\hline NDVI & - & - & $0,7047 * *$ \\
\hline $\mathrm{SAVI}_{\mathrm{L}=0,25}$ & $-0,7895^{* *}$ & $-0,8330 * *$ & - \\
\hline $\mathrm{SAVI}_{\mathrm{L}=0,50}$ & $-0,7895^{* *}$ & $-0,8331 * *$ & $0,7063^{* *}$ \\
\hline
\end{tabular}

**Correlação significativa a $1 \%$ de probabilidade.

Entretanto, o índice NDVI não apresentou correlação significativa nos povoamentos aos 4 e 18 anos. Esse comportamento pode ser atribuído a presença de sombreamento nesses talhões.

Buscando explicar essa relação com base na análise visual e nos dados mensurados a campo, observou-se que aos 4 anos, os povoamentos apresentam grande densidade populacional em função da ausência de desbaste. A Prática do desbaste visa a redução do número de indivíduos, e sua ausência nesses povoamentos desencadeou pontos de sombreamento nos estratos inferiores e por vezes, em indivíduos adjacentes. Esse comportamento está associado as características de reflexão das folhas sombreadas, as quais segundo Ponzoni (2002), possuem menores espaços de ar na estrutura interna, reduzindo a reflectância da REM nessa faixa espectral.

Aos 18 anos, os dados de campo exibiram crescimento dissimilar, apresentando diferenças significativas na altura dos indivíduos dentro das UAs, uma vez que os povoamentos foram originados por sementes. Desse modo, mesmo em relevo plano, esses povoamentos apresentaram pontos de sombreamento decorrentes de diferentes estratos. A partir da existência de diferentes estratos horizontais, os indivíduos dominantes projetam suas copas acima de uma cota média do dossel, acarretando sombreamento daquelas posicionadas a baixo (Ponzoni, 2002).

No entanto, aos 23 anos, os povoamentos apresentaram comportamento distinto dos anteriores, sendo parte atribuída às práticas de manejo realizadas e parte aos fenômenos naturais ocorridos ao longo do tempo. Desse modo, esses povoamentos caracterizam-se por maior espaçamento entre as copas e pela presença de falhas no dossel, o que permitiu maior influência do plano de fundo na resposta espectral, evidenciando uma relação direta entre o volume e os dados espectrais.

As variáveis selecionadas pelo método Stepwise no processo de modelagem do volume para povoamentos de E. grandis estão expostas na Tabela 3. Entretanto, povoamentos aos 18 e 23 anos não apresentaram modelos com coeficiente de determinação $\left(\mathrm{R}^{2}{ }_{\text {aj }}\right)$ superior a 0,70 , assim não foram demonstrados nesse estudo.
Observou-se que aos 4 anos de idade o modelo tendo como variáveis independentes o índice $\mathrm{SAVI}_{\mathrm{L}=0,50} \mathrm{e}$ $\mathrm{SAVI}_{\mathrm{L}=0,25}$ melhor respondeu a variabilidade do volume de madeira estocado, apresentando ajuste na reta de regressão de 0,74. Para esse mesmo modelo, os parâmetros estatísticos compostos pelo erro padrão da estimativa $\left(\mathrm{S}_{\mathrm{yx}}\right)$ e Índice de Furnival (IF) demonstraram um valor de $60,08 \mathrm{~m}^{3} /$ ha e $91,90 \mathrm{~m}^{3} /$ ha, respectivamente.

A validação do modelo que melhor descreveu o volume aos 4 anos de idades pelo teste do Qui-quadrado $\left(\mathrm{X}^{2}\right)$ demonstrou que os valores de $\mathrm{X}^{2}$ calculados foram inferiores ao valor de $\mathrm{X}^{2}$ tabelado. Assim, é possível inferir que não houve diferença significativa entre os valores reais e estimados.

Goergen (2014) ao modelar o volume com imagens TM obteve $\mathrm{R}^{2}$ de 0,48 para povoamentos de E. dunnii aos 5 anos, já para E. urograndis aos 2,2 anos obteve um $\mathrm{R}^{2}$ de 0,57 e 0,45 aos 4,2 anos.

Pacheco et al. (2012) ao utilizar dados espectrais do satélite Landsat 5/TM na caracterização do dossel de Eucalyptus spp obteve melhores respostas com a banda B5. Assim, essa variável compôs o modelo de estimativa do DAP, altura total e densidade de plantas, sendo que esses modelos explicaram $61 \%, 50 \%$ e $68 \%$ da variabilidade dos dados, respectivamente.

Hall et al. (2006) ao estimar o volume para povoamentos de coníferas baseando-se em modelos de altura e fechamento de copa, obtiveram $\mathrm{R}^{2}$ aj de 0,65 a partir das bandas 3, 4 e 5 do Landsat 7/ETM+. Esse modelo demonstrou um erro na estimativa do volume para a floresta temperada de $70,3 \mathrm{~m}^{3} / \mathrm{ha}$ ao utilizar dados espectrais. Thenkabail et al. (2003) utilizando dados do sensor IKONOS e Landsat 7/ETM+ para estimar o volume florestal encontraram valores de $\mathrm{R}^{2}$ aj que variaram de 0,34 a 0,69 .

Canavesi et al. (2010) obteve melhores resultados na associação de dados hiperespectrais com o volume florestal considerando os povoamentos situados em áreas pouco iluminadas, desse modo o $\mathrm{R}_{\text {aj }}^{2}$ para os modelos em tais condições variou de 0,589 a 0,704 . 
Tabela 3. Modelos com maior ajuste para os povoamentos estudados.

\begin{tabular}{cccccc}
\hline Idade & Equação & $\begin{array}{c}\mathrm{S}_{\mathrm{yx}} \\
\left(\mathrm{m}^{3} / \mathrm{ha}\right)\end{array}$ & $\mathrm{R}^{2}{ }_{\text {aj }}$ & $\mathrm{IF}$ & $\mathrm{F}$ \\
\hline $\begin{array}{c}4 \\
\text { anos }\end{array}$ & $\mathrm{V}=-178132-159905 * \ln \left(\mathrm{SAVI}_{\mathrm{L}=0,50}\right)+215267 *$ & 60,08 & 0,74 & 91,90 & $28,12^{* *}$ \\
$\left(\mathrm{SAVI} \mathrm{L}_{\mathrm{L}=0,25}\right)$ & $\mathrm{V}=-809,5-2318,6 * \ln \left(\mathrm{SAVI}_{\mathrm{L}=0,25}+57636,8 * \mathrm{~B} 2\right.$ & 64,00 & 0,70 & 97,90 & $23,77 * *$ \\
\hline
\end{tabular}

**Significativo a $1 \%$ de probabilidade; $\mathrm{V}=$ volume de madeira $\left(\mathrm{m}^{3} / \mathrm{ha}\right) ; \ln =$ logaritmo natural; Syx $=$ erro padrão da estimativa $\left(\mathrm{m}^{3} / \mathrm{ha}^{2}\right) \mathrm{R}^{2}$ aj $=$ coeficiente de determinação ajustado; $\mathrm{IF}=$ Índice de Furnival; $\mathrm{F}=$ valor de significância; $\mathrm{B} 2$ = reflectância da banda do azul; $\mathrm{SAVI}=0,25=$ reflectância do índice $\mathrm{SAVI}_{\mathrm{L}=0,25} ; \mathrm{SAVI}_{\mathrm{L}=0,50}=$ reflectância do índice $\mathrm{SAVI} \mathrm{L}_{\mathrm{L}=0,50}$.

Estudos demonstram a influência de fatores intrínsecos da área de estudo, como a qualidade do sítio, clima e solo na acurácia das estimativas, quando utilizado dados espectrais para a modelagem dos parâmetros biofísicos (HOLMGREN et al., 2000; MENG et al., 2007). Assim, considera-se adequado para cada área de estudo e para cada sensor, ajustar um modelo de estimativa, desse modo, consideram-se as limitações ocasionadas pela dependência da cena e sítio.

No presente estudo, os erros contidos nos modelos de estimativa de volume de E. grandis, tendem a ser decorrentes do modo com que as UAs foram identificadas a campo. As UAs foram localizadas a partir do conhecimento prévio de seu posicionamento utilizando GPS modelo Garmin Etrex Legend $\AA$, o qual apresenta erro de aproximadamente $10 \mathrm{~m}$, sendo essa precisão, influenciada pelo fechamento do dossel florestal.

Do mesmo modo, estudos que relacionam dados orbitais com parâmetros biofísicos da vegetação são influenciados pela geometria de iluminação do terreno (CANAVESI et al., 2010). A área de estudo em questão caracteriza-se por apresentar um relevo plano, variando de 5 a $15 \mathrm{~m}$ acima do nível do mar.

Neste sentido, esse fator pode ter contribuído positivamente para o ajuste dos modelos de estimativas do presente estudo, uma vez que isolou a interferência da geometria de iluminação nos valores de radiância armazenados pelo sensor.

\section{CONCLUSÕES}

A diferenciação dos estágios de crescimento da espécie E. grandis foi possível por meio dos dados espectrais extraído do sensor OLI/Landsat 8. Assim, imagens OLI/Landsat 8 são eficientes para o planejamento e manejo adequado da atividade florestal permitindo agilidade e maior eficiência na sua produção.

A maioria dos indicadores utilizados apresentaram sensibilidade na estimativa do volume de madeira. Entretanto, a variável espectral representada pelo índice SAVI mostrou englobar melhor as variações do volume em campo para povoamentos comerciais de E. grandis.

\section{REFERÊNCIAS}

AARDT, J. A. N. V.; NORRIS-ROGERS, M. Spectral age interactions in managed, even-aged Eucalyptus plantations: application of discriminant analysis and classification and regression trees approaches to hyperspectral data. International Journal of Remote Sensing, 29. n. 6, p. 1841-1845, 2008.

Bernardes, S. Índices de vegetação e valores de proporção na caracterização de floresta tropical primária e estádios sucessionais na área de influência da Floresta Nacional de Tapajós - Estado do Pará. 1998. 86 p. Dissertação (Mestrado em Sensoriamento Remoto) - Instituto Nacional e Pesquisas Espaciais, 1998.

BERRA, E. F. Avaliação e comparação de imagens LISS III/ResourceSAT-1 e TM/LANDSAT 5 para estimar volume de madeira de um plantio de Pinus elliottii. 2013. 127 p. Dissertação (Mestrado em Sensoriamento Remoto) - Universidade Federal do Rio Grande do Sul, 2013.

CANAVESI, V.; PONZONI, F. J.; VALERIANO, M. M. Estimativa de volume de madeira em plantios de Eucalyptus spp. utilizando dados hiperespectrais e dados topográficos. Revista Árvore, v. 34, n. 3, p. 539-549, 2010.

CARREIRAS, J. M. B.; PEREIRA, J. M. C.; PEREIRA, J. $\mathrm{S}$. Estimation of tree canopy cover in evergreen oak woodlands using remote sensing. Forest Ecology and Management, v. 223, n. 3, p. 45-53, 2006.

CASSOL, H. L. G. Estimativa de biomassa e estoque de carbono em um fragmento de floresta ombrófila mista com uso de dados ópticos de sensores remotos. 2013. 143 p. Dissertação (Mestrado em Sensoriamento Remoto) - Universidade Federal do Rio Grande do Sul, 2013.

COSTA, L. F.; ALBA, E.; MELLO, E. P.; SILVA, E. A.; TRAMONTINA, J.; PEREIRA, R. S. Estimativa do volume e densidade de árvores em povoamentos de Eucalyptus grandis a partir de dados espectrais. In: SIMPÓSIO LATINO AMERICANO DE MANEJO FLORESTAL, 6., 2015, Santa Maria, RS. Anais... Santa Maria, SIMANEJO, v.1, p. 210-217, 2015. 
DUBE, T.; MUTANGA, O. 2015. Evaluating the utility of medium-spatial resolution Landsat 8 multispectral sensor in quantifying aboveground biomass in uMgeni Catchment, South Africa. Journal of Photogrammetry and Remote Sensing, v. 101, n. 1, p. 36-46, 2015.

FERRAZ, A. S.; SOARES, V. P.; SOARES, C. P. B.; RIBEIRO, C. A. A. S., BINOTI, D. H. B.; LEITE, H.G. Estimativa de estoque de biomassa em um fragmento florestal usando imagens orbitais. Floresta e ambiente, v. 21, n. 3, p. 286-296, 2014.

GOERGEN, L. C. G. Uso da reflectância de imagens Landsat 5 TM na identificação de plantios de Eucalyptus dunnii e Eucalyptus urograndis e sua correlação com o volume de madeira. 2014. 100 p. Dissertação (Mestrado em Engenharia Florestais) Universidade Federal de Santa Maria, 2014.

HALL, R. J.; SKAKUN, R. S.; ARSENAULT, B. S.; CASE, B. S. Modeling forest stand structure attributes using Landsat ETM+ data: Application to mapping of aboveground biomass and stand volume. Forest Ecology and Management, v. 225, n. 3, p. 378-390, 2006.

HOLMGREN, J.; JOYCE, S.; NILSSON, M.; OLSSON, H. Estimating Stem Volume and Basal Area in Forest Compartments by Combining Satellite Image Data with Field Data. Scandinavian Journal of Forest Research, v. 15, n. 1, p. 103-111, 2000.

IBÁ. Indústria Brasileira de Árvores. Relatório Ibá 2017. Brasília, 2017. Disponível em: $<$ http://iba.org/pt/biblioteca-iba/publicacoes>. Acesso em: 15 set. 2017.

IBGE. Instituto Brasileiro de Geografia e Estatística. Levantamento sistemático da produção agrícola. 2017. Disponível em: <http://www.ibge.gov.br/home/estatistica/indicadores/a gropecuaria/lspa/lspa_201708_1.shtm>. Acesso em: 15 set. 2017.

JENSEN, J. R. Sensoriamento remoto do ambiente: uma perspectiva em recursos terrestres. São José dos Campos, Parêntese, 2009.

MACIEL, M. N. M. Estimativa de parâmetros estruturais de uma floresta primária na Amazônia Oriental através de dados orbitais. 2002. 144 f. Tese (Doutorado em Ciências Florestais) Universidade Federal do Paraná, 2002.

MENG, Q.; CEISZEWSKI, C. J.; MADDEN, M.; BORDES, B. A linear mixed-effects model of biomass and volume of trees using Landsat ETM+ images. Forest Ecology and Management, v. 244, n. 3, p. 93-101, 2007.
MOHAMMADI, J.; JOIBARY, S. S.; YAGHMAEE, F.; MAHINY, A. S. Moddeling forest stand volume and density using Landsat ETM+ data. International Journal of Remote Sensing, v. 31, n. 11, p. 2959-2975, 2010.

PACHECO, L. R. F.; PONZONI, F. J., SANTOS, S. B.; ANDRADES-FILHO, C. O.; MELLO, M. P.; CAMPOS, R. C. Structural characterization of canopies of Eucalyptus spp. using radiometric data from TM/Landsat 5. Cerne, v. 18 , n. 1, p. $105-116,2012$.

PONZONI, F. J.; PACHECO, L. R. F.; SANTOS, S. B.; ANDRADES-FILHO, C. O. Caracterização espectrotemporal de dosséis de Eucalyptus spp. mediante dados radiométricos TM/Landsat5. Cerne, v. 2, n. 2, p. 267-275, 2015.

PONZONI, F. J., SHIMABUKURO, Y. E.; KUPLICH, T. M. Sensoriamento remoto aplicado ao estudo da vegetação. 2 . ed. São José Dos Campos, Saraiva, 2012.

PONZONI, F. J. Sensoriamento remoto no estudo da vegetação: diagnosticando a mata atlântica. In: RUDORFF, B. F. T.; MORAES, E. C.; PONZONI, F. J.; CAMARGO JÚNIOR, H.; CONFORTE, J. C.; MOREIRA, J. C.; EPIPHANIO, J. C. N.; MOREIRA, M.A.; KAMPEL, M.; ALBUQUERQUE, P. C. G.; MARTINI, P. R.; FERREIRA, S. H.; TAVARES JÚNIOR, S. S.; SANTOS, V. M. N. (Ed.). Curso de uso de sensoriamento remoto no estudo do meio ambiente. 2002. São José dos Campos: INPE, 22 p.

PUHR, G.B.; DONOGHUE, D.N.M. Remote sensing of upland conifer plantations using Landsat TM data: a case study from Galloway, south-west Scotland. International Journal of Remote Sensing, v. 21, n. 4, p. 633-646, 2000.

R DEVELOPMENT CORE TEAM. R: A Language and Environment for Statistical Computing. Vienna: R Foundation for Statistical Computing. 2016. Available online at: $<$ http://www.R-project.org/>.

SCHNEIDER, P. R.; SCHNEIDER, P. S. P.; SOUZA, C. A. M. Análise de regressão aplicada à Engenharia Florestal. 2. ed. Santa Maria: Facos, 2009.

TILLACK, A., CLASEN, A., KLEINSCHMIT, B., FÖRSTER, M. Estimation of the seasonal leaf area index in an alluvial forest using high-resolution satellite-based vegetation indices. Remote Sensing of Environment, v. 141, v. 1, p. 52-64, 2014.

THENKABAIL, P. S.; ENCLONA, E. A.; ASHTON, M. S.; LEGG, C.; DIEU, M. J. Hyperion, Ikonos, Ali and ETM+ sensors in the study of African rainforests. Remote Sensing of Environment, v. 90, n. 1, p. 23-43, 2004. 